ARTICLE

\title{
Exploring atomic defects in molybdenum disulphide monolayers
}

Jinhua Hong ${ }^{1, \star}$, Zhixin $\mathrm{Hu}^{2, \star}$, Matt Probert ${ }^{3}, \mathrm{Kun} \mathrm{Li}^{4}$, Danhui $\mathrm{Lv}^{1}$, Xinan Yang ${ }^{5}$, Lin $\mathrm{Gu}^{5}$, Nannan Mao ${ }^{6,7}$, Qingliang Feng ${ }^{6}$, Liming $\mathrm{Xie}^{6}{ }^{6}$, Jin Zhang ${ }^{7}$, Dianzhong Wu ${ }^{8}$, Zhiyong Zhang ${ }^{8}$, Chuanhong Jin ${ }^{1}$, Wei $\mathrm{Ji}^{2,9}$, Xixiang Zhang ${ }^{4}$, Jun Yuan ${ }^{1,3} \&$ Ze Zhang ${ }^{1}$

Defects usually play an important role in tailoring various properties of two-dimensional materials. Defects in two-dimensional monolayer molybdenum disulphide may be responsible for large variation of electric and optical properties. Here we present a comprehensive joint experiment-theory investigation of point defects in monolayer molybdenum disulphide prepared by mechanical exfoliation, physical and chemical vapour deposition. Defect species are systematically identified and their concentrations determined by aberration-corrected scanning transmission electron microscopy, and also studied by ab-initio calculation. Defect density up to $3.5 \times 10^{13} \mathrm{~cm}^{-2}$ is found and the dominant category of defects changes from sulphur vacancy in mechanical exfoliation and chemical vapour deposition samples to molybdenum antisite in physical vapour deposition samples. Influence of defects on electronic structure and charge-carrier mobility are predicted by calculation and observed by electric transport measurement. In light of these results, the growth of ultra-high-quality monolayer molybdenum disulphide appears a primary task for the community pursuing high-performance electronic devices.

\footnotetext{
${ }^{1}$ State Key Laboratory of Silicon Materials, Key Laboratory of Advanced Materials and Applications for Batteries of Zhejiang Province, School of Materials Science and Engineering, Zhejiang University, Hangzhou, Zhejiang 310027, China. ${ }^{2}$ Beijing Key Laboratory of Optoelectronic Functional Materials and MicroNano Devices, Department of Physics, Renmin University of China, Beijing 100872, China. ${ }^{3}$ Department of Physics, University of York, Heslington, York YO10 5DD, UK. ${ }^{4}$ Advanced Nanofabrication, Imaging and Characterization Core Lab, King Abdullah University of Science and Technology (KAUST), Thuwal 239955, Kingdom of Saudi Arabia. ${ }^{5}$ Instituteof Physics, Chinese Academy of Sciences, c/o Collaborative Innovation Center of Quantum Matter, Beijing 100190, China. ${ }^{6}$ CAS Key Laboratory of Standardization and Measurement for Nanotechnology, National Center for Nanoscience and Technology, Beijing 100190, China. ${ }^{7}$ Center for Nanochemistry, Beijing National Laboratory for Molecular Sciences, Key Laboratory for the Physics and Chemistry of Nanodevices, State Key Laboratory for Structural Chemistry of Unstable and Stable Species, College of Chemistry and Molecular Engineering, Peking University, Beijing 100871, China. ${ }^{8}$ Key Laboratory for the Physics and Chemistry of Nanodevices and Department of Electronics, Peking University, Beijing 100871, China. ${ }^{9}$ Department of Physics and Astronomy, Collaborative Innovation Center of Advanced Microstructures, Shanghai Jiao Tong University, Shanghai 200240, China. ${ }^{\star}$ These authors contributed equally to this work. Correspondence and requests for materials should be addressed to C.J. (email: chhjin@zju.edu.cn) or to W.J. (email: wji@ruc.edu.cn) or to J.Y. (email: jun.yuan@york.ac.uk).
} 
T he success of graphene $e^{1,2}$ offers a paradigm for the exploration of novel low-dimensional physical phenomena ${ }^{3}$ and physical properties in two-dimensional (2D) crystal systems ${ }^{4,5}$. However, its intrinsic shortcoming lies in its zero bandgap, which strongly hinders its application in logical electronic devices. Among the post-graphene development, 2D semiconducting molybdenum disulphide and other transition metal dichalcogenides ${ }^{6,7}$ have recently appeared on the horizon of materials science and condensed matter physics. Monolayer $\mathrm{MoS}_{2}$ is a direct-gap semiconductor that exhibits a substantially improved efficiency in its photoluminescence ${ }^{8,9}$. Valley polarization occurs due to significant spin-orbit coupling and leads to optical circular dichroism ${ }^{10-12}$ in the monolayer system. The electronic transport of $\mathrm{MoS}_{2}$-based field effect transistors (FETs) shows steep sub-threshold swing of $70 \mathrm{mV} \mathrm{dec}^{-1}$ (refs 13-15) and a high on/off ratio up to $10^{8}$ (ref. 16). A metalinsulator transition happens when carrier densities reaches $10^{13} \mathrm{~cm}^{-2}$, which also increases the effective mobility ${ }^{17-19}$. Owing to its unique optical and electric properties, $\mathrm{MoS}_{2}$ is believed to be a promising candidate as a building block for future applications in nanoelectronics and optoelectronics ${ }^{6}$.

Wafer-scale production of atomically thin layers is paramount for $\mathrm{MoS}_{2}$ to be used as a candidate channel material for electronic and optoelectronic devices ${ }^{13-19}$. Among the currently available preparation methods, mechanical exfoliation (ME) is deemed less efficient for these large-scale applications, even though it produces the highest-quality samples exhibiting the best electric performance. Physical and chemical vapour deposition ${ }^{17,20-25}$ methods are more compatible for the scalable growth of high-quality samples. However, the experimentally attainable mobility is still one or two order-of-magnitude lower than the theoretical value of $410 \mathrm{~cm}^{2} \mathrm{~V}^{-1} \mathrm{~s}^{-1}$ (refs 13,18,26-29). For back-gated $\mathrm{m}-\mathrm{MoS}_{2}$ FET devices, the highest mobility reported so far reaches $81 \mathrm{~cm}^{2} \mathrm{~V}^{-1} \mathrm{~s}^{-1}$ for ME sample $\mathrm{c}^{28}, 45 \mathrm{~cm}^{2} \mathrm{~V}^{-1} \mathrm{~s}^{-1}$ for chemical vapour deposition (CVD) ${ }^{17}$ and $<1 \mathrm{~cm}^{2} \mathrm{~V}^{-1} \mathrm{~s}^{-1}$ for physical vapour deposition (PVD $)^{25}$. The major scattering mechanism for the mobility deterioration has been recently suggested as due to the presence of plentiful localized band tail states ${ }^{30}$ caused by short-range disordered structural defects (such as vacancies ${ }^{28,31}$ and grain boundaries ${ }^{32}$ ), and Coulomb traps ${ }^{33,34}$. However, the roles played by various defects in electric and optoelectronic properties are yet to be explicitly understood. There have been few investigations reported on point defects, mostly vacancies, and grain boundaries in $\mathrm{m}-\mathrm{MoS}_{2}$ (refs 28,30-32,35,36). These studies are, however, often performed with samples made by preparatory methods, for example, ME or CVD, which essentially limits the scope of those studies.

Here we present a systematic investigation of the point defects in distinctly prepared $\mathrm{m}-\mathrm{MoS}_{2}$ by combining atomically resolved annular dark-field scanning transmission electron microscopy (ADF-STEM) imaging, density functional theory (DFT) calculation and electric transport measurements. We observe, for the first time, that antisite defects with molybdenum replacing sulphur are dominant point defects in PVD-grown $\mathrm{MoS}_{2}$, while the sulphur vacancies are predominant in $\mathrm{ME}$ and CVD specimens. These experimental observations are further supported qualitatively by the growth mechanism and quantitatively by the defects' formation energies calculations. The DFT calculations, in addition, predict the electronic structures and magnetic properties of $\mathrm{m}-\mathrm{MoS}_{2}$ with antisite defects. We also discuss the influence of defects on the phonon-limited carrier mobility theoretically, and further examine them by electric transport in defective $\mathrm{m}-\mathrm{MoS}_{2}$-based FETs. Our systematic investigation of point defects, especially antisites, will further deepen our understanding of this novel $2 \mathrm{D}$ atomically thin semiconductor and pave the way for the scalable electronic application of the family of atomically thin transition metal chalcogenides.

\section{Results}

Statistics of point defects. For the purpose of the analysis of defects and their concentration, we have chosen about ten samples prepared under the optimized fabrication condition (see the Methods section and Supplementary Note 1 for the details of sample synthesis) from each method (ME, PVD and CVD) and then transferred each sample onto at least two TEM grids independently for ADF-STEM characterizations. The crystalline quality and the choice of samples for statistical analysis are presented in Supplementary Figs 1-9. Figure 1 summarized the most significant results obtained from our analysis on these $\mathrm{MoS}_{2}$ samples. For the PVD specimen, antisite defects with one Mo atom replacing one or two $\mathrm{S}$ atoms $\left(\mathrm{Mos}_{\mathrm{S}}\right.$ or $\mathrm{Mo}_{\mathrm{S2}}$ ) are frequently observed, marked with red dashed circles shown in Fig. 1a, while the dominant defects for the ME and CVD samples are $S$ vacancies with one $\left(V_{S}\right)$ or two $\left(V_{S 2}\right) S$ atoms absent, as marked by green dashed circles in Fig. 1b. As the STEM's $Z$-contrast mechanism ${ }^{37}$, that is, $I \sim Z^{1.6-2.0}$ ( $I$ and $Z$ are the image contrast and atomic number, respectively), predicts, Mo and $S$ atoms can be unambiguously discriminated, with Mo $(Z=44)$ showing bright contrast and two superposed $S$ atoms showing dim contrast in the lattice of $\mathrm{m}-\mathrm{MoS}_{2}$. Following a similar argument and quantitative image analysis, various defects, for example, $\mathrm{Mo}_{S}$ or $\mathrm{V}_{\mathrm{S}}$ where the lattice image presents abnormal intensity variation, can be clearly identified individually through direct imaging and their atomic structures further verified by ab-initio calculations (please refer to Supplementary Table 1 for details of each atomic defect).

We show the relative importance of each type of point defects in Fig. 1c,d. Figure 1c presents the total counts of different point defects based on over 70 atomically resolved ADF-STEM images for each type, that is, ME, PVD or CVD MoS 2 samples. It is found that the dominant type of point defects in each sample highly depends on the specific sample preparation method. The $V_{S}$ vacancy is the predominant point defects in $\mathrm{ME}$ and CVD samples, with its concentration of about $(1.2 \pm 0.4) \times 10^{13} \mathrm{~cm}^{-2}$ (Supplementary Fig. 6), close to the results reported previously ${ }^{30}$. Atomic defects $\mathrm{V}_{\mathrm{Mo}}$ (one Mo atom missing) and $\mathrm{S}_{\mathrm{Mo}}$ (one $\mathrm{S}$ atom replacing Mo site) were also found, but with much lower concentrations as shown in Fig. 1c. In contrast, the histogram also shows that antisite defects $\mathrm{Mo}_{\mathrm{S} 2}$ and $\mathrm{Mo}_{\mathrm{S}}$ are dominant in PVD samples, with their concentrations higher than that of $\mathrm{V}_{\mathrm{S}}$. The density of $\mathrm{Mos}_{\mathrm{S} 2}$ and $\mathrm{Mos}_{\mathrm{S}}$ reaches $(2.8 \pm 0.3) \times 10^{13}$ and $7.0 \times 10^{12} \mathrm{~cm}^{-2}$, corresponding to an atomic percent of $0.8 \%$ and $0.21 \%$, respectively (counted on the total number of all Mo and S atoms). Such a defect concentration is surprisingly high if the defects were regarded as impurity doping, which is usually only achieved in degenerate semiconductor ${ }^{38}$ (for instance $10^{-2} \sim 10^{-4}$ ). It is, therefore, of vital importance to understand how they modify the electronic properties of $\mathrm{m}-\mathrm{MoS}_{2}$ as elucidated below.

Structural characterization of point defects. So far, there have been few reports concerning the structures of sulphur vacancies ${ }^{31,39}$ and their impacts on the electronic transport properties ${ }^{30,40}$ of $\mathrm{m}-\mathrm{MoS}_{2}$; by contrast, there is still a lack of detailed knowledge on the antisite defects, which is at such an unexpected high doping level in PVD samples. Hence, we focus more on antisite defects. In Fig. 2a-e, we highlight all the images of the experimentally observed antisite defects in $\mathrm{m}-\mathrm{MoS}_{2}$ (see also Supplementary Fig. 10), which can be 

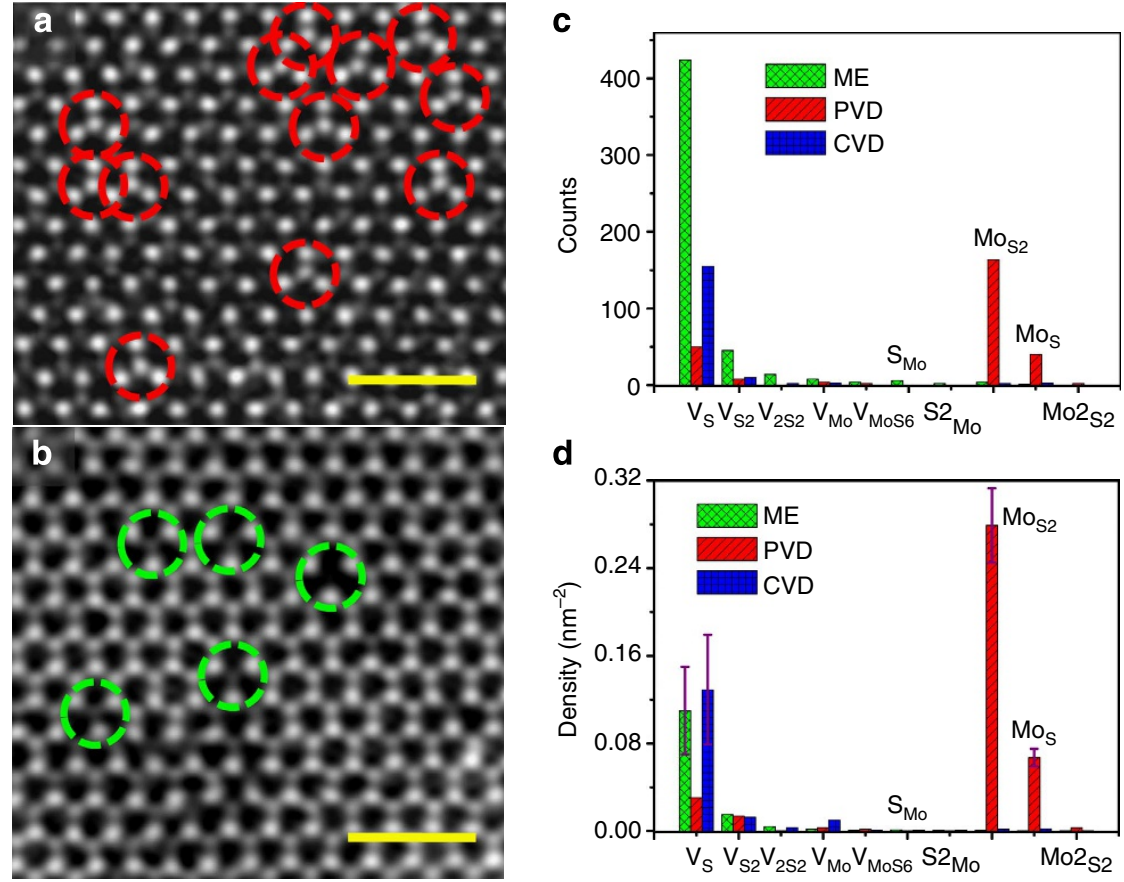

Figure 1 | Atomic resolved STEM-ADF images to reveal the distribution of different point defects. (a) Antisite defects in PVD MoS 2 monolayers. Scale bar, $1 \mathrm{~nm}$. (b) Vacancies including $\mathrm{V}_{\mathrm{S}}$ and $\mathrm{V}_{\mathrm{S} 2}$ observed in ME monolayers, similar to that observed for CVD sample. Scale bar, $1 \mathrm{~nm}$. (c,d) Histograms of various point defects in PVD, CVD and ME monolayers. Error estimates are given for the dominant defects (more details on the statistics can be found in Supplementary Fig. 6). ME data are in green, PVD data in red and CVD in blue.

grouped into two categories. One is the antisite defects with Mo atom(s) substituting $\mathrm{S}$ atom(s), including $\mathrm{Mo}_{\mathrm{S}}, \mathrm{Mo}_{\mathrm{S} 2}$ and $\mathrm{Mo} 2_{\mathrm{S} 2}$ (Fig. $2 \mathrm{a}-\mathrm{c}$ ). The other category is the antisite defects with $\mathrm{S}$ atom occupying the site of Mo, namely $\mathrm{S}_{\mathrm{Mo}}$ and $\mathrm{S} 2_{\mathrm{Mo}}$ (Fig. $2 \mathrm{~d}, \mathrm{e}$ ). The experimental identification of these antisite defects can be further unambiguously supported by the quantitative image simulation based on DFT-predicted atomic structures of all antisite defects. The fully relaxed DFT-predicted atomic structures of antisite defects were shown in Fig. $2 \mathrm{k}-\mathrm{t}$, where the atomic displacement and structure deformation are explicitly observable, especially for antisites $\mathrm{Mo}_{\mathrm{S} 2}$ and $\mathrm{S} 2_{\mathrm{Mo}}$. Associated side views of these relaxed structures are available in Fig. 2p-t. The ADF image simulations (Fig. 2f-j) based on the calculated structures fit quite well with the experimental images shown in Fig. 2a-e, respectively, especially for the off-centre feature observable in antisites $\mathrm{Mo}_{\mathrm{S} 2}$ and $\mathrm{S} 2_{\mathrm{Mo}}$, which can be tentatively attributed to Jahn-Teller distortions.

Energetics of predominant point defects in different $\mathbf{m}-\mathrm{MoS}_{2}$. The distribution of different atomic defects in $\mathrm{m}-\mathrm{MoS}_{2}$ certainly depends on their preparation process. A full exploration of the growth dynamics requires a comprehensive experiment-theory joint investigation, which is beyond the scope of the present work, although it is of fundamental interest. Here we provide a qualitative explanation, based on our DFT calculations, to reveal the microscopic physical mechanism of the preparation-processdependent defect formation. The formation energy $\left(\Delta E_{\mathrm{Form}}\right)$ for all the point defects are calculated and summarized in Table 1 , as consistent with a previous report ${ }^{35}$. Here, chemical potentials of elements $\mathrm{Mo}$ and $\mathrm{S}$ were employed to calculate the formation enthalpy $\left(\Delta H_{\text {Form }}\right)$ of defects. To account for a range of different possible reservoirs (for example, bulk element or bulk $\mathrm{MoS}_{2}$ ), each enthalpy is given with a range, as listed in Table 1. Two widely used DFT codes (CASTEP ${ }^{41}$ and VASP (Vienna Ab-initio Simulation Package ${ }^{42}$ ) are adopted to exploit the full range of functionality available and demonstrate the consistency of the calculated results in Table 1.

An ME sample is exfoliated from $\mathrm{MoS}_{2}$ natural mineral. After the $\mathrm{MoS}_{2}$ mineral was formed and/or extracted, either element $\mathrm{S}$ or Mo of $\mathrm{MoS}_{2}$ is prone to reach a solid-gas phase equilibrium. Owing to a higher saturated vapour pressure of $S$, the mineral-form $\mathrm{MoS}_{2}$ has to release more $\mathrm{S}$ than Mo atoms into the gas phase and thus $S$ is prone to be deficient in $\mathrm{MoS}_{2}$. Reflecting this fact, vacancies $V_{S}$ and $V_{S 2}$ have the lowest $\Delta E_{\text {Form }}$ of $2.12 \mathrm{eV}$ and $4.14 \mathrm{eV}$, respectively, among all the defects. The formation energies of all the antisite defects are higher than $5 \mathrm{eV}$, indicating that the $\mathrm{S}$-deficient mineral-form $\mathrm{MoS}_{2}$ favours the formation of $\mathrm{S}$ vacancies, leading to the observation of the most common defect of $V_{S}$, followed by $\mathrm{V}_{\mathrm{S} 2}$, and almost no antisite defect in ME samples.

In a typical PVD process, $\mathrm{MoS}_{2}$ precursor is sublimated into the gas phase with clusters and atoms, carried by Ar gas (mixed with $\mathrm{H}_{2}$ ), and then condensed into a solid-phase $\mathrm{MoS}_{2}$. Sulphur has a larger saturated vapour pressure so that more $S$ atoms in the gas phase will leave the preparation chamber, thus establishing a S-deficient and Mo-rich condition. These clusters and atoms are highly mobile and are thus prone to form an ordered structure of $\mathrm{MoS}_{2}$ in the lowest total energy. Considering $n+1$ Mo and $2 n-1 \mathrm{~S}$ atoms for example, they have two options, namely, forming (i) $n$ $\mathrm{MoS}_{2}$ units with one $\mathrm{Mos}_{\mathrm{S}}$ antisite or (ii) $n+1 \mathrm{MoS}_{2}$ units with three $\mathrm{V}_{\mathrm{S}}$ vacancies. The exact value of $n$ does not affect the energetic difference between these two types of defects. We thus arbitrarily instantiate $n$ as 107 , namely $108 \mathrm{Mo}$ and $213 \mathrm{~S}$ atoms in total. The total energy of the antisite option is $-1,774.83 \mathrm{eV}$, while that for the vacancy case is $-1,774.26 \mathrm{eV}$ which is $0.57 \mathrm{eV}$ less stable than the former. A similar relation also applies to antisite defect $\mathrm{Mo}_{\mathrm{S} 2}$ with an energy gain of $0.92 \mathrm{eV}$. A sample with one antisite $\mathrm{Mo}_{\mathrm{S}}$ or $\mathrm{Mo}_{\mathrm{S} 2}$ shares the same number of Mo and $\mathrm{S}$ atoms with another sample that has three or four $\mathrm{S}$ vacancies, respectively. The formation energies of antisites $\mathrm{Mo}_{\mathrm{S}}$ and $\mathrm{Mo}_{\mathrm{S} 2}$ were, therefore, divided by three and four, respectively, to make 

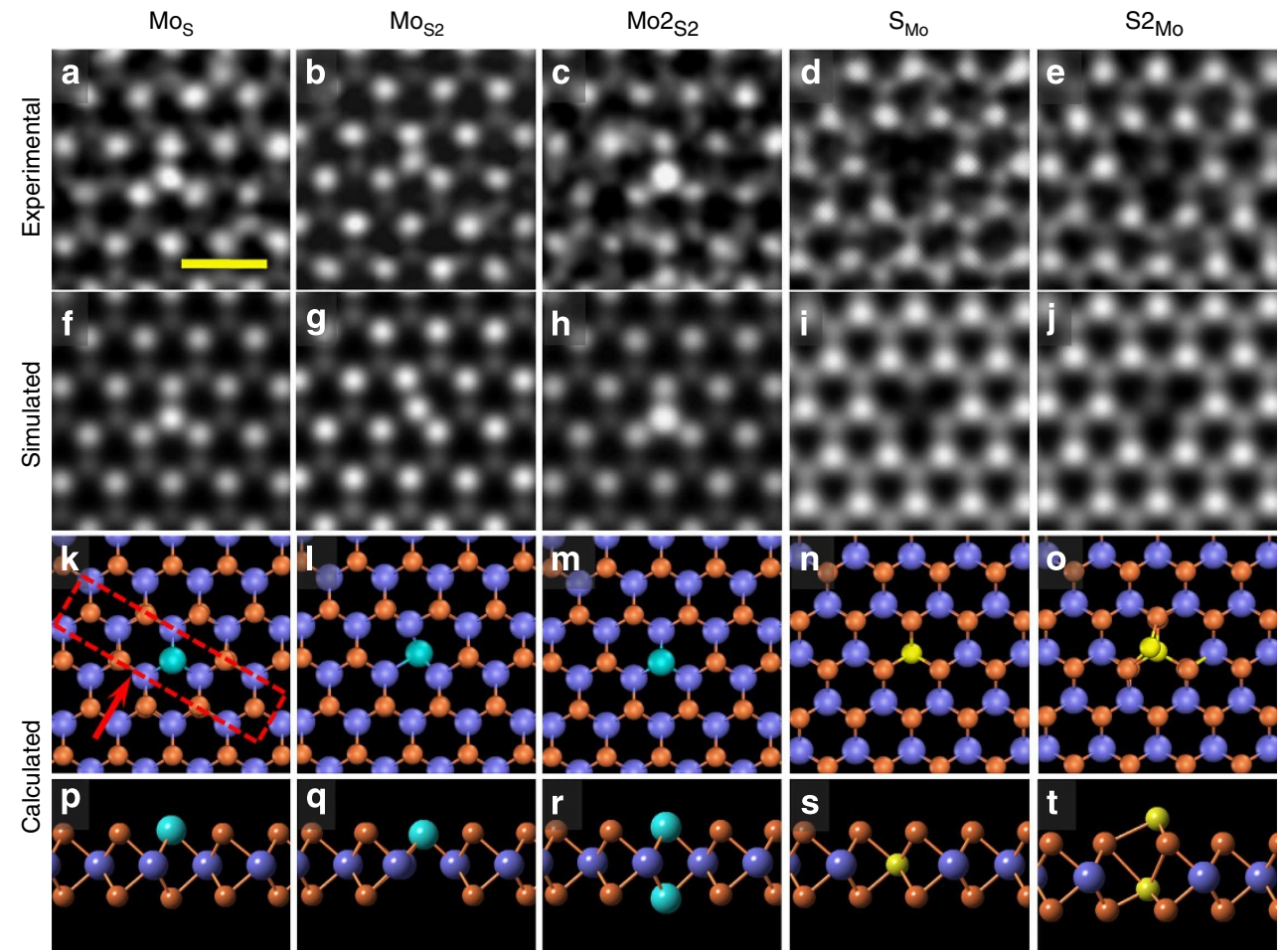

Figure 2 | Atomic structures of antisite defects. (a-c) High-resolution STEM-ADF images of antisite Mos, Mos2 and Mo2 $2_{S 2}$, respectively. The former two antisites (highlighted by the red dashed rectangle in $\mathbf{k}$ ) are dominant in PVD-synthesized $\mathrm{MoS}_{2}$ single layers. Scale bar, $0.5 \mathrm{~nm}$ (d,e) Atomic structures of antisite defects $\mathrm{S}_{\mathrm{Mo}}$ and $\mathrm{S} 2_{\mathrm{Mo}_{0}}$, respectively. (f-j) Simulated STEM images based on the theoretically relaxed structures of the corresponding point defects in (a-e), using simulation software QSTEM ${ }^{49}$. (k-t) Relaxed atomic model of all antisite defects in a-e through DFT calculation, with top and side views, respectively. Light blue, Mo atoms; gold, S atoms. For ease of comparison, we have presented the simulated ADF images before the atomistic schematics of the DFT calculated structures.

Table 1 | Formation energy $\left(\Delta E_{\text {Form }}\right)$ and enthalpy $\left(\Delta H_{\text {Form }}\right)$ of considered point defects.

\begin{tabular}{|c|c|c|c|}
\hline & \multirow{2}{*}{$\begin{array}{c}\text { CASTEP } \\
\Delta H_{\text {Form }}(\mathrm{eV})\end{array}$} & \multicolumn{2}{|c|}{ VASP } \\
\hline & & $\Delta H_{\text {Form }}(\mathrm{eV})$ & $\Delta E_{\text {Form }}(\mathrm{eV})$ \\
\hline$\overline{M o s}$ & $6.22 \sim 7.29$ & $5.45 \sim 6.09$ & 5.79 \\
\hline $\mathrm{Mo}_{\mathrm{S} 2}$ & 11.15 & 7.95 & 7.54 \\
\hline $\mathrm{M}_{\mathrm{O} 2 \mathrm{~s} 2}$ & - & $9.81 \sim 11.09$ & 10.49 \\
\hline $\mathrm{S}_{\mathrm{Mo}}$ & $6.65 \sim 5.58$ & $6.11 \sim 5.47$ & 5.77 \\
\hline $\mathrm{S} 2_{\text {мо }}$ & 8.00 & 7.09 & 7.49 \\
\hline$V_{S}$ & $2.74 \sim 1.67$ & $2.86 \sim 2.22$ & 2.12 \\
\hline $\mathrm{V}_{\mathrm{S} 2}$ & - & $5.63 \sim 4.34$ & 4.14 \\
\hline$V_{\text {Mo }}$ & $6.98 \sim 4.84$ & $7.28 \sim 5.99$ & 6.20 \\
\hline
\end{tabular}

CASTEP, Cambridge Sequential Total Energy Package; VASP, Vienna Ab-initio Simulation Package.

The formation enthalpy is defined as $\Delta H_{\text {Form }}=E_{\text {Defect }}-E_{\text {Pure }}+n \times \mu_{\text {Removed }}-m \times k \mu_{\text {Added. }} \mu$ is the chemical potential of the removed and/or added atom to form a defect, while the formation

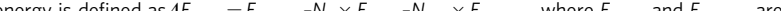
 single atom energy of Mo and $S$ in more details).

Different exchange-correlation functionals are used in the VASP and CASTEP codes as discussed in the text.

these energies quantitatively comparable with a single S vacancy, as required by the comparison with Boltzmann distribution. We have renormalized $\Delta E_{\text {Form }}^{\prime}\left(\mathrm{Mo}_{\mathrm{S}}\right)=1.93 \mathrm{eV}, \Delta E_{\text {Form }}^{\prime}\left(\mathrm{Mo}_{\mathrm{S} 2}\right)=$ $1.89 \mathrm{eV}$, which give rise to a ratio of $p\left(\mathrm{Mo}_{\mathrm{S} 2}\right): p\left(\mathrm{Mo}_{\mathrm{S}}\right): p\left(\mathrm{~V}_{\mathrm{S}}\right)=$ 10.5:6.7:1 at the growth temperature of $1,100 \mathrm{~K}$. This ratio is comparable with the experimental probability density ratio of $\mathrm{Mo}_{\mathrm{S} 2}: \mathrm{Mo}_{\mathrm{S}}: \mathrm{V}_{\mathrm{S}}=9: 2.3: 1$ in PVD samples.

The CVD process is distinctly different from ME or PVD. Extra $\mathrm{S}$ vapour is supplied to replace $\mathrm{O}$ in the $\mathrm{MoO}_{3}$ precursor under an S-rich condition. We suspect that there are small amount of residual $\mathrm{O}$ atoms taking the position of $\mathrm{S}$ atoms in the resulting $\mathrm{MoS}_{2}$ sheets, due to the competition between Mo-O and $\mathrm{Mo}-\mathrm{S}$ bonding in the reaction chamber. Our $a b$-initio calculation, not shown here, suggests that these $\mathrm{O}$ atoms are $1.99 \mathrm{eV}$ less stable than corresponding $2 \mathrm{~S}$ and usually tend to desorb into the gas phase leaving vacancies at the $S$ sites, that is, $S$ vacancies. It is argued that Mo atoms may jump into the $S$ vacancies and form Mo antisites. Despite of the S-rich condition, even if Mo is rich in a certain local environment, Mo atoms may be firmly bonded with oxygen in the precursor, which strongly limits the diffusion of Mo, making the formation of Mo antisite from mobile Mo atom and $S$ vacancy much less likely.

Electronic structures. The electronic structure of point defects plays a crucial role in determining the electric properties of these defective $\mathrm{m}-\mathrm{MoS}_{2}$. Vacancy $\mathrm{V}_{\mathrm{S}}$ and its effect on electronic structures have been recently reported ${ }^{30,35}$; we thus focus on the less-studied antisite defects (please refer to Supplementary Fig. 11 for our $\mathrm{ADF}$ imaging and DFT calculation of $\mathrm{V}_{\mathrm{S}}$ ). Figure 3 shows the theoretically predicted band structures and projected densityof-states of two primary antisites $\mathrm{Mo}_{\mathrm{S}}$ and $\mathrm{Mo}_{\mathrm{S2}}$. Our results give a bandgap of $1.73 \mathrm{eV}$ for a defect-free $\mathrm{m}-\mathrm{MoS}_{2}$ (Supplementary Fig. 12), close to the experimentally observed optical bandgap of $1.8 \mathrm{eV}$ (ref. 8). Defect states with nearly flat band dispersion for $\mathrm{Mo}_{\mathrm{S} 2}$ and $\mathrm{Mo}_{\mathrm{S}}$ reside inside the band gap of a perfect $\mathrm{m}-\mathrm{MoS}_{2}$ (Fig. 3a,d). These states mostly comprises the $d$ orbitals of four Mo atoms around the defect. In addition, the orbital hybridization of $\mathrm{Mo}$ and $\mathrm{S}$ atoms results in extended wavefunctions involving the surrounding atoms, forming a 'superatom' with a radius of roughly $6 \AA$, as shown in Fig. 3b,c,f.

Magnetic properties of $\mathrm{m}-\mathrm{MoS}_{2}$ have not been reported yet, as it is believed to be a non-magnetic material. Nevertheless, we did 
a

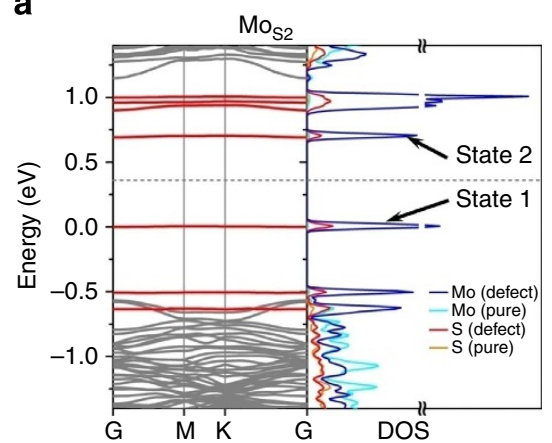

d

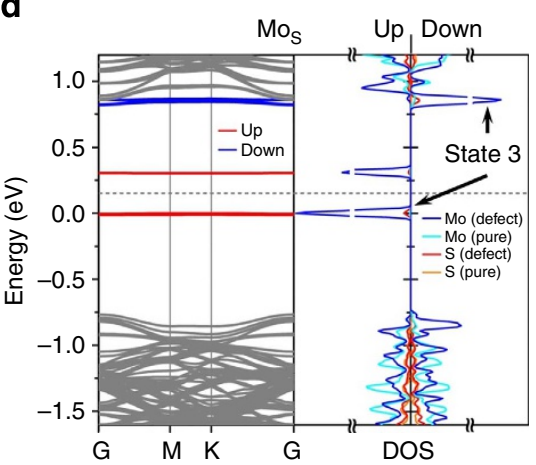

b

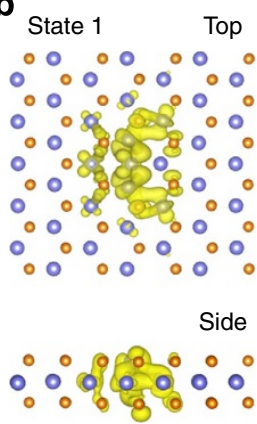

e

$\mathrm{Mo}_{\mathrm{S}}$ total

- 000

$\circ 000.000$

000 8 800

- octó 0 . 0

$\circ .0$ \%8.0

$00.8,00$

$00^{\circ} \circ 0^{\circ} \circ 0^{\circ}$

Side

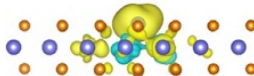

c

State 2 Top

$\circ \circ \circ \circ \circ \circ \circ$

- 00.0000

$\circ \circ 0^{\circ} 00^{\circ} 00^{\circ}$

$\circ 0$ ces 00

0.0.0.

$\circ .80000$

00.800.

00.00000

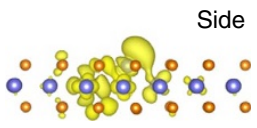

f

State 3 Top

$\circ \circ \circ \circ \circ 0^{\circ} \circ$

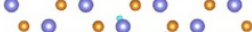

$0.0008,00$

$\circ \circ \circ 000$

0.000000

0.0080 .0

000000000

$\circ \circ \circ .000$

Side

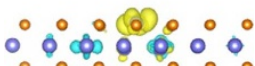

Figure 3 | Electronic properties of predominant antisite defects in $\mathbf{M o S}_{\mathbf{2}}$ monolayer. (a) Band structure and corresponding density of states (DOS) of antisite defect $\mathrm{Mo}_{\mathrm{s} 2}$. The grey bands are from normal lattice sites, similar to conduction band and valence band of perfect monolayer, while the discrete red bands show the localized defects states. The DOS is projected onto the atoms around the defect (defect) and those in the middle plane of two adjacent defects (pure), respectively. The grey dash line indicates the position of the Fermi Level. (b,c) Real-space distribution of the wave functions of the two defect states below and above the Fermi energy. (d) The band structure and DOS of antisite $\mathrm{Mo}_{\mathrm{S}}$, with a similar colour scheme of $\mathbf{a}$, but the two spin components are coloured in red (spin-up) and blue (spin-down), respectively. (e) Spin density of antisite Mos, defined as $\rho_{\text {up }}-\rho_{\text {down }}$ charge densities $\rho_{\text {up }}$ and $\rho_{\text {down }}$ are spin-resolved for spin-up and -down components, which are represented by yellow and blue isosurfaces, respectively. (f) Spin-resolved real-space distribution of the wave function of the two marked defect states (State 3 ) in $\mathbf{d}$. The isosurface value in $\mathbf{b}, \mathbf{c}, \mathbf{e}, \mathbf{f}$ is $0.001 e$ Bhor ${ }^{-3}$.

find a local magnetic moment of $2 \mu_{\mathrm{B}}$ in antisite $\mathrm{Mo}_{\mathrm{S}}$, while the values for other defects, for example, $\mathrm{V}_{\mathrm{S}}, \mathrm{V}_{\mathrm{S} 2}$ and $\mathrm{Mo}_{\mathrm{S} 2}$, are smaller than $0.1 \mu_{\mathrm{B}}$, and hence are negligible. The magnetic moment of $2 \mu_{\mathrm{B}}$ for a Mos antisite is not localized only on the central Mo atom, with the surrounding atoms contributing roughly $20 \%$ of the total moment probably due to the strong hybridization among these atoms in a 'superatom', as shown in the visualized total spin density (Fig. 3e). Detailed distribution of magnetic moment is available in Supplementary Fig. 13. The spin-resolved real-space distribution of a defect-induced state (state 3) marked in Fig. 3d was plotted in Fig. 3f to illustrate the origin of the magnetism. The occupied spin-up component (yellow isosurface) is mainly composed of the $d_{\mathrm{xy}}$ and $d_{\mathrm{x} 2-\mathrm{y} 2}$ orbitals of the antisite Mo atom, while the unoccupied spin-down component (cyan isosurface) is projected onto the $d_{\mathrm{xy}}$ and $d_{\mathrm{z} 2}$ orbitals of surrounding Mo atoms, consistent with the total spin charge density shown in Fig. 3e. More detailed discussion on the magnetic property of antisites are presented in Supplementary Fig. 14 and Supplementary Note 2.

Carrier mobility in defective samples. As there have been plentiful reports on the transport of ME $\mathrm{MoS}_{2}$-based FETs with electron mobility $1 \sim 81 \mathrm{~cm}^{2} \mathrm{~V}^{-1} \mathrm{~s}^{-1}$ (refs $15,16,18,26,28,43,44$ ), we focus on the transport properties of CVD and PVD monolayers. Figure $4 \mathrm{a}-\mathrm{d}$ presents the output and transfer characteristics of fabricated FETs based on PVD and CVD $\mathrm{MoS}_{2}$, respectively. Our transport measurements (in Fig. $4 \mathrm{a}-\mathrm{d}$ ) of defective $\mathrm{MoS}_{2}$-based FETs reveal that the PVD and CVD $\mathrm{MoS}_{2}$ has electron mobility 0.5 and $11 \mathrm{~cm}^{2} \mathrm{~V}^{-1} \mathrm{~s}^{-1}$, respectively. All these results are well comparable with the reported mobilities of $\mathrm{m}-\mathrm{MoS}_{2}$ of $1 \sim 81 \mathrm{~cm}^{2} \mathrm{~V}^{-1} \mathrm{~s}^{-1}$ for $\mathrm{ME}^{15,16,18,26,28,43,44}$, $5 \sim 45 \mathrm{~cm}^{2} \mathrm{~V}^{-1} \mathrm{~s}^{-1}$ for $\mathrm{CVD}^{17,29}$ and the reported values of $<1 \mathrm{~cm}^{2} \mathrm{~V}^{-1} \mathrm{~s}^{-1}$ for PVD m-MoS $($ ref. 25) respectively.

Theoretically, we focus on the effect of the defects on the phonon-limited carrier mobilities ${ }^{45-47}$. Table 2 lists the calculated effective masses, deformation potentials and estimated mobilities derived based on the predicted electron mobility of $410 \mathrm{~cm}^{2} \mathrm{~V}^{-1} \mathrm{~s}^{-1}$ in a perfect $\mathrm{m}-\mathrm{MoS}_{2}$ (ref. 48). $\mathrm{MoS}_{2}$ samples are usually n-type, we thus primarily focus on the electron mobility. It is found that the phonon-limited mobility of electrons flowing in the intrinsic conduction band is, exceptionally, nearly unaffected by the presence of vacancies $\left(\mathrm{V}_{\mathrm{S}}\right.$ or $\left.\mathrm{V}_{\mathrm{S} 2}\right)$, but reduced by three times in the samples with antisite defects, whereas the phonon-limited mobility of holes carried by the intrinsic valence band is more sensitive to these defects and reduces roughly three times for vacancy and more than four times for antisite. Both vacancy and antisite are strong electron-scattering centres that the mobility derived from the defect states ( $d-e$ and $d-h$ ) for either electron or hole is fairly small, mostly smaller than 1 and $10 \mathrm{~cm}^{2} \mathrm{~V}^{-1} \mathrm{~s}^{-1}$, respectively. The defect states strongly affect, but not overwhelmingly dominate, the overall carrier mobility of the samples, owing to the relative low density of defects and the strongly localized defect states. On the other hand, in a real FET device the measured mobility can be affected by the contact resistance ${ }^{45}$ or the carrier density ${ }^{19}$. Furthermore, the trapped charges would act as a scattering centre ${ }^{15,40}$. A hopping transport caused by localized disorder is also observed ${ }^{30,33}$. Both can 
a

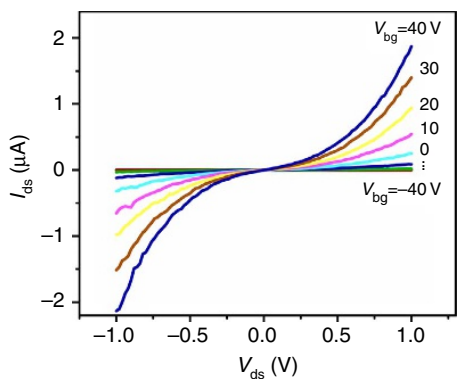

C

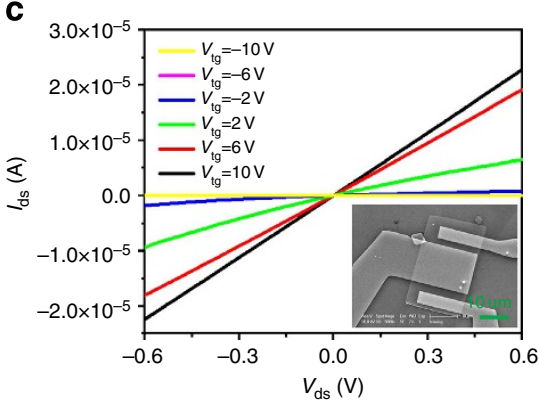

b

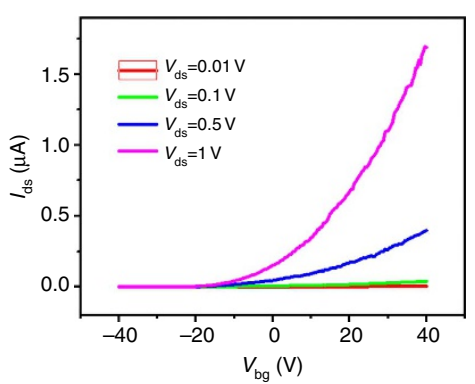

d

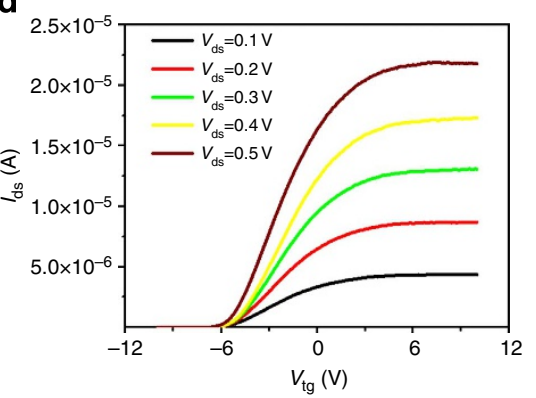

Figure 4 | Electric transport of defective $\mathbf{M o S}_{\mathbf{2}} \cdot(\mathbf{a}, \mathbf{b})$ Output and transfer characteristics of PVD MoS 2 -based FET. (c,d) Output and transfer characteristics of CVD $\mathrm{MoS}_{2}$-based FET.

Table 2 | Phonon-limited carrier mobility estimation of perfect and defective $\mathrm{MoS}_{\mathbf{2}}$ monolayers.

\begin{tabular}{|c|c|c|c|c|c|c|c|c|c|c|c|c|}
\hline & \multicolumn{4}{|c|}{$\mathbf{m}^{\star} / \mathbf{m}_{\mathbf{0}}$} & \multicolumn{4}{|c|}{$E(e V)$} & \multicolumn{4}{|c|}{$\mu\left(\mathrm{cm}^{2} \mathrm{v}^{-1} \mathrm{~s}^{-1}\right)$} \\
\hline & e & $\mathbf{h}$ & d-e & d-h & e & $\mathbf{h}$ & d-e & d-h & e & $\mathbf{h}$ & d-e & d-h \\
\hline Perfect & 0.40 & -0.57 & - & - & -14.9 & -3.4 & - & - & 410 & 3850 & - & - \\
\hline$V_{S}$ & 0.43 & -0.96 & 35.2 & 105.6 & -13.9 & -3.4 & -4.5 & 5.4 & 410 & 1390 & $<1$ & $<1$ \\
\hline $\mathrm{V}_{\mathrm{S} 2}$ & 0.42 & -1.2 & 31.1 & 9.2 & -13.9 & -3.1 & -4.9 & 6.8 & 426 & 1066 & $<1$ & 4 \\
\hline $\mathrm{Mos}_{\mathrm{S}}$ & 0.99 & -1.2 & 75.5 & 34.1 & -11.1 & -4.0 & -3.0 & -2.9 & 123 & 631 & $<1$ & 2 \\
\hline $\mathrm{Mos}_{\mathrm{S} 2}$ & 0.71 & -1.1 & 8.8 & 28.6 & -13.3 & -3.6 & -4.3 & -6.8 & 164 & 902 & 10 & $<1$ \\
\hline
\end{tabular}

Carrier types ' $e$ ' and ' $h$ ' denote 'electron' and 'hole' for the original conduction and valence bands in perfect MoS $_{2}$ monolayer, and ' $d-e^{\prime}$ and ' $d-h$ ' represent those for the defect states, respectively. $m^{\star}$ is carrier effective mass and $E$ is the deformation potential. All mobilities are estimated based on the value for the electron mobility in the perfect monolayer of $410 \mathrm{~cm}^{2} V{ }^{-1} \mathrm{~s}^{-1}$ (ref. 48 ). The change of elastic moduli of perfect and defective samples is so small, which can be safely neglected. Each defective monolayer consists of only one type of defects (either vacancy or antisite) in a $6 \times 6$ supercell,

close to an equivalent defect density revealed in the statistics.

effectively reduce the mobility of the device. Nevertheless, our theory shows that the measured mobility is, most likely, correlated with the primary type of defects in a sample.

\section{Discussion}

We have to address the possible influence of electron beam irradiation on the formation of atomic defects and to distinguish the native from irradiation-induced defects. The observed antisite defects are believed to be native, not caused by electron beam irradiation. We envisage that two steps are involved in the formation of a $\mathrm{Mo}_{\mathrm{S}}$ defect from a well-prepared sample, namely the formation of a $V_{S}$ vacancy and Mo adatom, followed by the capture of the Mo adatom by the $S$ vacancy. Although sulphur vacancy could be created by electron beam sputtering ${ }^{31,39}$, the formation of a Mo adatom and adjacent Mo vacancy need substantially high energy transfer from electron irradiation, which is less likely. The in-situ experiments show that the Mo adatoms are very mobile, but rarely jump into $S$ vacancies to form antisite defects (Supplementary Note 3 and Supplementary Figs 15 and 16). On considering these experimental and simulation results, the observed $\mathrm{Mo}_{\mathrm{S}}$ and $\mathrm{Mo}_{\mathrm{S} 2}$ antisites should be confidently regarded as intrinsic defects. In terms of $\mathrm{S}$ vacancies, as suggested by early studies $^{30,31}$, the concentration of sulphur vacancies may be slightly overestimated due to beam damage even if the microscope works at low accelerating voltage (Supplementary Fig. 17).

$\mathrm{MoS}_{2}$ sheets are extensively adopted in electronic devices. These point defects, as localized disorders, are significant scattering centres of carriers, which may reduce the mobility of charge carriers through the intrinsic conduction or valence band, especially for samples with antisite defects. Therefore, growth of ultra-high-quality $\mathrm{m}-\mathrm{MoS}_{2}$ is of crucial importance to fabricate high-performance electronic devices. On the other side, the presence of defects may provide us novel routes to tailor the properties of $\mathrm{m}-\mathrm{MoS}_{2}$. We predicted theoretically for the first time that antisite $\mathrm{Mo}_{\mathrm{S}}$ shows a magnetic moment of $2 \mu_{\mathrm{B}}$. Our prediction of local magnetic moments may promote further investigations on the magnetic properties of defective $\mathrm{MoS}_{2}$ monolayers. Given the strong optoelectronic response of $\mathrm{MoS}_{2}$ layers with $\mathrm{Mo}_{\mathrm{S}}$ defects, it is a probable material that is capable for optical manipulation of local magnetic moment. If the defect density goes sufficiently high, it may expect an appreciably large magnetic exchange interaction between defects, and thus become a promising model system for the studies of dilute-magnetic semiconductors and $2 \mathrm{D}$ magnetism.

Based on these findings, we propose an application-oriented strategy for fabricating atomically thin $\mathrm{MoS}_{2}$. In terms of electric 
applications, extra $\mathrm{S}$ should be introduced into the growth process or post-growth treatment, to restrain the formation of antisite defects for PVD specimen or to heal the abundant $S$ vacancies for CVD and ME specimens, whereas in respect to magnetism, varied pressure of $S$ in the PVD growth could produce different densities of $\mathrm{Mo}_{\mathrm{Sx}}$ antisites that remain to be explored for magnetic applications.

In summary, our systematic investigation of geometric and electronic structures of antisites and vacancies of $\mathrm{m}-\mathrm{MoS}_{2}$ by ADFSTEM imaging and DFT calculation has led to a considerable progress in our understanding of the variation of the electric and magnetic properties induced by these point defects. We have demonstrated that minimizing point defects, especially antisites, is paramount for electric transport applications, while controllably introduced antisites may produce atomic size local magnetic moments. All these results considerably improve the understanding of point defect in atomically thin transition metal dichalcogenides and should benefit their potential applications in optoelectronic and nanoelectronic devices.

\section{Methods}

Sample preparations and transfer. $\mathrm{ME} \mathrm{m}-\mathrm{MoS}_{2}$ was prepared by micro-cleavage $^{8}$ of natural bulk crystal (SPI Supplies) using scotch tapes. The monolayer was identified from the optical contrast of thin flakes under an optical microscope (Zeiss $\mathrm{A} 2 \mathrm{~m}$ ) and then transferred onto copper TEM grids covered with holey carbon films.

CVD monolayers were synthesized through the reduction of precursor $\mathrm{MoO}_{3}$ by sulphur vapour flow at ambient pressures following the previously reported method $^{21,32}$. PVD $\mathrm{MoS}_{2}$ monolayers used in this study were synthesized by thermal evaporation of $\mathrm{MoS}_{2}$ powders (Sigma-Aldrich, 99\%) at a temperature of $950^{\circ} \mathrm{C}$. Ar (2 s.c.c.m.) and $\mathrm{H}_{2}$ (0.5 s.c.c.m.) were used as the carriers gases, following the reported method in ref. 25. The pressure of the growth chamber was about $8 \mathrm{~Pa}$ and the growth time was usually $10 \mathrm{~min}$. In terms of sample synthesis, the advantages and disadvantages of these methods and liquid-phase exfoliation are compared in Supplementary Table 2.

PVD and CVD $\mathrm{MoS}_{2}$ monolayers were transferred onto the TEM grid as follows: first, the $\mathrm{SiO}_{2}$ substrates with monolayer samples were covered with polymethyl methacrylate (PMMA) film after spin coating and then dried in air at $120^{\circ} \mathrm{C}$ for $5 \mathrm{~min}$. The substrates were immersed into the boiling sodium hydroxide solution $\left(1 \mathrm{moll}^{-1}\right)$, which was heated up to $200^{\circ} \mathrm{C}$ to etch away the underneath $\mathrm{SiO}_{2}$ layers. The floating PMMA film was picked up with a clean glass slide and then transferred into the distilled water for several cycles to wash away surface residues. In the next step, the PMMA film was lifted out by a TEM grid covered with lacey carbon film and then dried naturally in ambient. This TEM grid was heated at $120^{\circ} \mathrm{C}$ for $5 \mathrm{~min}$ in air before immersion into hot acetone for about $24 \mathrm{~h}$, to remove the PMMA. Before the ADF-STEM characterization, all the monolayer specimens on TEM grids were annealed at $200{ }^{\circ} \mathrm{C}$ in air for $10 \mathrm{~min}$ to reduce surface residues and/or contaminations.

STEM characterization and image simulation. Most of the structural characterizations of $\mathrm{m}-\mathrm{MoS}_{2}$ were carried out with a probe-corrected Titan ChemiSTEM (FEI, USA). We operated this microscope at an acceleration voltage of $80 \mathrm{kV}$ to alleviate specimen damage induced by beam radiation. A low probe current was selected $(<70 \mathrm{pA})$ and the convergence angle was set to be $22 \mathrm{mrad}$. Under such a condition, the probe size was estimated to be close to $1.5 \AA$. To enhance the contrast of the sulphur sublattices, the so-called medium-range ADF mode rather than the high-angle ADF mode was used by adjusting the camera length properly. Some experiments (such as Figs $1 \mathrm{a}$ and $2 \mathrm{a}-\mathrm{c}$ ) were done with an ARM 200CF (JEOL, Japan), equipped with a cold field-emission gun. The advantage of higher energy resolution $(0.3 \mathrm{eV})$ and smaller probe size $(<1.2 \AA)$ provides higher resolution, thus giving rise to sharper contrast of the atomic images. All the experimental images shown in the main text and Supplementary Information were filtered through the standard Wiener deconvolution to partially remove the background noise for a better display (Supplementary Fig. 10).

It should be noted that intrinsic adatom defects were seldom observed experimentally and, therefore, they are not considered here. All the image statistics were done on the clean regions of the examined samples by ADF-STEM. On considering the good homogeneity of these samples prepared under the optimized conditions (see Supplementary Information), it shall not lead any large variations in the analysed defect population.

STEM-ADF image simulations of relaxed antisite defects were done by software QSTEM $^{49}$. The input parameters were set according to the experimental conditions. Probe size, convergence angle and acceptance angle of the ADF detector are critical and accounted for in the image simulation.
DFT calculations. The defect formation enthalpy for the first column of Table 1 was calculated using the total energy method with the plane-wave pseudopotential DFT code CASTEP ${ }^{41}$. The basic methodology is well known and has been widely used before for defect calculations. In this study, the Perdew-Burke-Ernzerhofgeneralized gradient approximation ${ }^{50}$ with ultrasoft potentials is used, as supplied in the CASTEP library. In addition, the dispersion interactions were added using the semi-empirical scheme of Grimme ${ }^{51}$. Structural optimizations of both ionic positions and cell vectors are performed using a modified Broyden-FletcherGoldfarb-Shanno-like scheme. The calculations were performed in a slab geometry of a $6 \times 6$ supercell of $\mathrm{m}-\mathrm{MoS}_{2}$ with a $15 \AA$ vacuum space in the $c$ axis direction perpendicular to the monolayer. A comparative study of the defect formation enthalpy and energy, together with the electronic and magnetic properties were also done by VASP simulation code ${ }^{42}$ using the same slab model. The projector augmented-wave method ${ }^{52}$ combined with a plane wave basis is adopted in the calculations. The energy cutoff for plane wave is $400 \mathrm{eV}$ in structural relaxation and increases to $500 \mathrm{eV}$ while calculating the energy and electronic properties. The optB86b exchange functional ${ }^{53}$ together with the vdW correlation ${ }^{54,55}$ was adopted for exchange-correlation functional. The Brillouin zone of the supercell is sampled by a $3 \times 3 \times 1 k$-mesh. All these structures are fully relaxed until the residual force for each atom is less than $0.02 \mathrm{eV} \AA^{-1}$.

Estimation of formation energy and enthalpy. The formation energy was defined as, $\Delta E_{\mathrm{Form}}=E_{\mathrm{System}}-N_{\mathrm{S}} \times E_{\mathrm{S} \_\mathrm{ML}}-N_{\mathrm{Mo}} \times E_{\mathrm{Mo} \_\mathrm{ML}}$, where $E_{\mathrm{S} \_\mathrm{ML}}=E_{\mathrm{S} \text { (single) }}$ $+E_{\mathrm{Bond}}, E_{\mathrm{Mo} \_\mathrm{ML}}=E_{\mathrm{Mo}(\text { single })}+2 E_{\mathrm{Bond}}$ and $E_{\mathrm{Bond}}=\left(E_{\mathrm{ML}}-E_{\mathrm{Mo}(\text { single })}-2 E_{\mathrm{S} \text { (single })}\right) / 3$ Formation enthalpy of defects is defined as $\Delta H_{\text {Form }}=E_{\text {Defect }}-E_{\text {Pure }}+n \times$ $\mu_{\text {Removed }}-m \times \mu_{\text {Added }}$, where $\mu_{\text {Removed }}$ and $\mu_{\text {Added }}$ are the chemical potentials of the removed and added atoms to form a defect, respectively. Chemical potentials of Mo and $\mathrm{S}$ in $\mathrm{MoS}_{2}$ fulfill the equation $\mu_{\mathrm{Mo}}+2 \mu_{\mathrm{S}}=\mu_{\mathrm{Mo}}^{*}+2 \mu_{\mathrm{S}}^{*}+\Delta H_{\mathrm{MoS}_{2}}$, where $\mu_{\mathrm{Mo}}^{*}$ is the chemical potential of Mo in the bulk form, $\mu_{\mathrm{S}}^{*}$ is the chemical potential of $\mathrm{S}$ in the $\alpha$-phase crystal form and $\Delta \mathrm{H}_{\mathrm{MoS}_{2}}$ is the formation enthalpy of $\mathrm{MoS}_{2}$. Although it is difficult to obtain the exact values of $\mu_{\mathrm{Mo}}$ and $\mu_{\mathrm{S}}$, the range of them can be deduced as $\mu_{\mathrm{Mo}}^{*}+\Delta H_{\mathrm{MoS}_{2}} \leq \mu_{\mathrm{Mo}} \leq \mu_{\mathrm{Mo}}^{*}, \mu_{\mathrm{S}}^{*}+\frac{1}{2} \Delta H_{\mathrm{MoS}_{2}} \leq \mu_{\mathrm{S}} \leq \mu_{\mathrm{S}}^{*}$.

There are two formation enthalpy values in Table 1 , the former one was computed by choosing $\mu_{\mathrm{Mo}}$ and $\mu_{\mathrm{S}}$ equal to $\mu_{\mathrm{Mo}}^{*}$ and $\mu_{\mathrm{S}}^{*}$, respectively, indicating that the removed (added) atoms come from (go to) the pure bulk form of Mo and S. For the latter value, we set $\mu_{\mathrm{Mo}}=\mu_{\mathrm{Mo}}^{*}+0.5 \times \Delta H_{\mathrm{MoS}_{2}}$ and $\mu_{\mathrm{s}}=\mu_{\mathrm{s}}^{*}+0.25 \times \Delta H_{\mathrm{MoS}_{2}}$. In this case, the source and drain of defect atoms are pure $\mathrm{MoS}_{2}$ ML. For antisite defects $\mathrm{Mo}_{\mathrm{S} 2}$ and $\mathrm{S} 2_{\mathrm{Mo}}$, both schemes give the same result.

Estimation of 'phonon-limited' carrier mobility. In 2D, the carrier mobility is given by the expression ${ }^{45-47}$

$$
\mu_{2 \mathrm{D}}=\frac{e \hbar^{3} C_{2 \mathrm{D}}}{k_{\mathrm{B}} T m_{\mathrm{e}}^{*} m_{\mathrm{d}}\left(E_{1}^{i}\right)^{2}}
$$

where $m_{\mathrm{e}}^{*}$ is the effective mass in the transport direction and $m_{d}$ is the average effective mass determined by $m_{d}=\sqrt{m_{x}^{*} m_{y}^{*}}$. The term $E_{1}$ represents the deformation potential constant of the valence-band maximum for holes or conduction-band minimum for electrons along the transport direction, defined by $E_{1}^{i}=\Delta V_{i} /\left(\Delta l / l_{0}\right)$. Here $\Delta V_{\mathrm{i}}$ is the energy change of the $i^{\text {th }}$ band under proper cell compression and dilatation, $l_{0}$ is the lattice constant in the transport direction and $\Delta l$ is the deformation of $l_{0}$.

FET fabrication and transport. The monolayer $\mathrm{MoS}_{2}$-based FET devices are fabricated through the following process. First, source (S) and drain (D) electrodes of the devices were defined via e-beam lithography and a 5/45 nm Ti/Au film was then evaporated followed by a standard lift-off process. In addition, the back-gated $\mathrm{MoS}_{2}$ FETs were then finished. Second, the top-gated devices were begun with forming gate insulator. Gate oxide layer $\left(30 \mathrm{~nm} \mathrm{HfO}_{2}\right.$ film) was grown under $90^{\circ} \mathrm{C}$ through Atomic Layer Deposition (Cambridge NanoTech Inc.). Lastly, the gate electrode window was also defined by e-beam lithography, followed by evaporation of $5 \mathrm{~nm}$ Ti and $45 \mathrm{~nm}$ Au thin film, and the top-gated $\mathrm{MoS}_{2}$ FETs are finished after a lift-off process. The as-fabricated devices were measured through Keithley 4200 semiconductor analyser on a probe station at room temperature and in air. An example of the device architecture is shown in Supplementary Fig. 18.

\section{References}

1. Novoselov, K. S. et al. Two-dimensional gas of massless Dirac fermions in graphene. Nature 438, 197-200 (2005).

2. Geim, A. K. \& Novoselov, K. S. The rise of graphene. Nat. Mater. 6, 183-191 (2007).

3. Zhang, Y. B., Tan, Y. W., Stormer, H. L. \& Kim, P. Experimental observation of the quantum Hall effect and Berry's phase in graphene. Nature 438, 201-204 (2005).

4. Novoselov, K. S. et al. Electronic properties of graphene. Phys. Stat. Sol. B 244, 4106-4111 (2007).

5. Li, X. et al. Large-area synthesis of high-quality and uniform graphene films on copper foils. Science 324, 1312-1314 (2009). 
6. Wang, Q. H., Kalantar-Zadeh, K., Kis, A., Coleman, J. N. \& Strano, M. S. Electronics and optoelectronics of two-dimensional transition metal dichalcogenides. Nat. Nanotechnol. 7, 699-712 (2012).

7. Ataca, C., Sahin, H. \& Ciraci, S. Stable single-layer $\mathrm{MX}_{2}$ transition-metal oxides and dichalcogenides in a honeycomb-like structure. J. Phys. Chem. C. 116, 8983-8999 (2012).

8. Mak, K. F., Lee, C., Hone, J., Shan, J. \& Heinz, T. F. Atomically thin $\mathrm{MoS}_{2}$ : a new direct-gap semiconductor. Phys. Rev. Lett. 105, 136805 (2010).

9. Splendiani, A. et al. Emerging photoluminesence in monolayer $\mathrm{MoS}_{2}$. Nano Lett. 10, 1271-1275 (2010).

10. Zeng, H., Dai, J., Yao, W., Xiao, D. \& Cui, X. Valley polarization in $\mathrm{MoS}_{2}$ monolayers by optical pumping. Nat. Nanotechnol. 7, 490-493 (2012).

11. Mak, K. F., He, K., Shan, J. \& Heinz, T. F. Control of valley polarization in monolayer $\mathrm{MoS}_{2}$ by optical helicity. Nat. Nanotechnol. 7, 494-498 (2012).

12. Cao, T. et al. Valley-selective circular dichroism of monolayer molybdenum disulphide. Nat. Commun. 3, 887 (2012).

13. Radisavljevic, B., Radenovic, A., Brivio, J., Giacometti, V. \& Kis, A. Single-layer $\mathrm{MoS}_{2}$ transistors. Nat. Nanotechnol. 6, 147-150 (2011).

14. Kim, S. et al. High-mobility and low-power thin-film transistors based on multilayer $\mathrm{MoS}_{2}$ crystals. Nat. Commun. 3, 1011 (2012).

15. Perera, M. M. et al. Improved carrier mobility in few-layer $\mathrm{MoS}_{2}$ field-effect transistors with ionic-liquid gating. ACS Nano 7, 4449-4458 (2013).

16. Zhang, Y. J., Ye, J. T., Matsuhashi, Y. \& Iwasa, Y. Ambipolar $\mathrm{MoS}_{2}$ thin flake transistors. Nano Lett. 12, 1136-1140 (2012).

17. Schmidt, H. et al. Transport properties of monolayer $\mathrm{MoS}_{2}$ grown by chemical vapor deposition. Nano Lett. 14, 1909-1913 (2014).

18. Zou, X. M. et al. Interface engineering for high-performance top-gated $\mathrm{MoS}_{2}$ field-effect transistors. Adv. Mater. 26, 6255-6261 (2014).

19. Radisavljevic, B. \& Kis, A. Mobility engineering and a metal-insulator transition in monolayer $\mathrm{MoS}_{2}$. Nat. Mater. 12, 815-820 (2013).

20. Wang, X., Feng, H., Wu, Y. \& Jiao, L. Controlled synthesis of highly crystalline $\mathrm{MoS}_{2}$ flakes by chemical vapor deposition. J. Am. Chem. Soc. 135, 5304-5307 (2013).

21. Lee, Y.-H. et al. Synthesis of large-area $\mathrm{MoS}_{2}$ atomic layers with chemical vapor deposition. Adv. Mater. 24, 2320-2325 (2012).

22. Liu, K.-K. et al. Growth of large-area and highly crystalline $\mathrm{MoS}_{2}$ thin layers on insulating substrates. Nano Lett. 12, 1538-1544 (2012).

23. Shi, Y. et al. van der Waals epitaxy of $\mathrm{MoS}_{2}$ layers using graphene as growth templates. Nano Lett. 12, 2784-2791 (2012).

24. Wu, S. et al. Vapor-solid growth of high optical quality $\mathrm{MoS}_{2}$ monolayers with near-unity valley polarization. ACS Nano 7, 2768-2772 (2013).

25. Feng, Q. et al. Growth of large-area $2 \mathrm{D} \operatorname{MoS}_{2(1-\mathrm{x})} \mathrm{Se}_{2 \mathrm{x}}$ semiconductor alloys. Adv. Mater. 26, 2648-2653 (2014).

26. Fuhrer, M. S. \& Hone, J. Measurement of mobility in dual-gated $\mathrm{MoS}_{2}$ transistors. Nat. Nanotechnol. 8, 146-147 (2013).

27. Radisavljevic, B. \& Kis, A. Measurement of mobility in dual-gated $\mathrm{MoS}_{2}$ transistors. Nat. Nanotechnol. 8, 147-148 (2013).

28. Yu, Z. et al. Towards intrinsic charge transport in monolayer molybdenum disulfide by defect and interface engineering. Nat. Commun. 5, 5290 (2014).

29. Liu, H. et al. Statistical study of deep submicron dual-gated field-effect transistors on monolayer chemical vapor deposition molybdenum disulfide films. Nano Lett. 13, 2640-2646 (2013).

30. Qiu, H. et al. Hopping transport through defect-induced localized states in molybdenum disulphide. Nat. Commun. 4, 2642 (2013).

31. Komsa, H.-P. et al. Two-dimensional transition metal dichalcogenides under electron irradiation: defect production and doping. Phys. Rev. Lett. 109, 035503 (2012)

32. Najmaei, S. et al. Vapour phase growth and grain boundary structure of molybdenum disulphide atomic layers. Nat. Mater. 12, 754-759 (2013).

33. Ghatak, S., Pal, A. N. \& Ghosh, A. Nature of electronic states in atomically thin $\mathrm{MoS}_{2}$ field-effect transistors. ACS Nano 5, 7707-7712 (2011).

34. Li, S.-L. et al. Thickness-dependent interfacial Coulomb scattering in atomically thin field-effect transistors. Nano Lett. 13, 3546-3552 (2013).

35. Zhou, W. et al. Intrinsic structural defects in monolayer molybdenum disulfide. Nano Lett. 13, 2615-2622 (2013).

36. van der Zande, A. M. et al. Grains and grain boundaries in highly crystalline monolayer molybdenum disulphide. Nat. Mater. 12, 554-561 (2013).

37. Pennycook, S. J. et al. Scanning Transmission Electron Microscopy-Imaging and Analysis (Springer, 2011).

38. Sze, S. M. \& Ng, K. K. Physics of Semiconductor Devices (John Wiley \& Sons, Inc., New Jersey, 2007).

39. Komsa, H. P., Kurasch, S., Lehtinen, O., Kaiser, U. \& Krasheninnikov, A. V. From point to extended defects in two-dimensional $\mathrm{MoS}_{2}$ : evolution of atomic structure under electron irradiation. Phys. Rev. B 88, 035301 (2013).

40. Zhu, W. et al. Electronic transport and device prospects of monolayer molybdenum disulphide grown by chemical vapour deposition. Nat. Commun. 5, 3087 (2014).

41. Clark, S. J. et al. First principles methods using CASTEP. Z. Kristallogr. 220, 567-570 (2005).

42. Kresse, G. \& Furthmuller, J. Efficient iterative schemes for $a b$ initio total-energy calculations using a plane-wave basis set. Phys. Rev. B 54, 11169-11186 (1996).
43. Baugher, B. W. H., Churchill, H. O. H., Yang, Y. \& Jarillo-Herrero, P. Intrinsic electronic transport properties of high-quality monolayer and bilayer $\mathrm{MoS}_{2}$. Nano Lett. 13, 4212-4216 (2013).

44. Bao, W. Z., Cai, X. H., Kim, D., Sridhara, K. \& Fuhrer, M. S. High mobility ambipolar $\mathrm{MoS}_{2}$ field-effect transistors: substrate and dielectric effects. Appl. Phys. Lett. 102, 042104 (2013).

45. Takagi, S.-i., Toriumi, A., Iwase, M. \& Tango, H. On the universality of inversion layer mobility in Si MOSFET's: part I-effects of substrate impurity concentration. IEEE Trans. Electr. Dev. 41, 2357-2362 (1994).

46. Qiao, J. S. et al. High-mobility transport anisotropy and linear dichroism in few-layer black phosphorus. Nat. Commun. 5, 4475 (2014).

47. Fiori, G. \& Iannaccone, G. Multiscale modeling for graphene-based nanoscale transistors. Proc. IEEE 101, 1653-1669 (2013).

48. Kaasbjerg, K., Thygesen, K. S. \& Jacobsen, K. W. Phonon-limited mobility in n-type single-layer $\mathrm{MoS}_{2}$ from first principles. Phys. Rev. B 85, 115317 (2012).

49. Koch, C. T. Determination of Core Structure Periodicity and Point Defect Density Along Dislocations. PhD thesis (Arizona State Univ., 2002).

50. Perdew, J. P., Burke, K. \& Ernzerhof, M. Generalized gradient approximation made simple. Phys. Rev. Lett. 77, 3865-3868 (1996).

51. Grimme, S. Semiempirical GGA-type density functional constructed with a long-range dispersion correction. J. Comput. Chem. 27, 1787-1799 (2006).

52. Blochl, P. E. Projector augmented-wave method. Phys. Rev. B 50, 17953-17979 (1994).

53. Klimes, J., Bowler, D. R. \& Michaelides, A. Van der waals density functionals applied to solids. Phys. Rev. B 83, 195131 (2011).

54. Dion, M., Rydberg, H., Schroder, E., Langreth, D. C. \& Lundqvist, B. I. Van der Waals density functional for general geometries. Phys. Rev. Lett. 92, 246401 (2004).

55. Lee, K., Murray, E. D., Kong, L., Lundqvist, B. I. \& Langreth, D. C. Higheraccuracy van der waals density functional. Phys. Rev. B 82, 081101 (2010).

\section{Acknowledgements}

This work was financially supported by the National Basic Research Program of China under grant numbers 2012CB932704, 2014CB932500 and 2015CB921000; National Science Foundation of China under grant numbers 51222202, 11004244, 11274380, 91433103 and 51472215; and the Fundamental Research Funds for the Central Universities under grant numbers 2014XZZX003-07 (ZJU), 12XNLJ03 and 14XNH062 (RUC). JY and MP acknowledge the EPSRC (UK) funding (EP/G070326, EP/J022098 and EP/K013564). WJ was supported by the Program for New Century Excellent Talents in Universities. The work on electron microscopy was mainly done at the Center for Electron Microscopy of Zhejiang University. VASP Calculations were performed at the Physics Lab of High-Performance Computing of Renmin University of China and Shanghai Supercomputer Center, and Castep calculations were done in York. JY acknowledges Pao Yu-Kong International Foundation for a Chair Professorship. We thank Zheng Meng, Haiyan Nan and Zhenhua Ni for their assistance on PL measurements, Fang Lin for her assistance on provding the codes for Wiener filtering, and Yanfeng Zhang and Liying Jiao for providing high-quality CVD $\mathrm{MoS}_{2}$ samples. We acknowledge Shengbai Zhang for his advice on formation mechanism of different defects and other calculations, and Wang Yao for his advices about the spin-valley effects in $\mathrm{m}-\mathrm{MoS}_{2}$.

\section{Author contributions}

J.H. and Z.H. contributed equally to this work. C.J., J.Y. and W.J. conceived the research. J.H., D.L. and N.M. contributed to the sample preparations. J.H. and C.J did most of the STEM characterizations, with the assistance from K.L., X.Y., L.G. and X.Z., J.H., J.Y. and C.J. were responsible for the STEM data analysis and image simulations. Z.H., W.J. and M.P. did the DFT calculations. N.M., L.X. and J.H. contributed to the synthesis, measurement and analysis of PL spectra and the PVD devices of PVD samples. D.W. and Z.Z. contributed to the FET device fabrications and electric transport measurements on CVD samples. All authors discussed the results and contributed to the preparation of the manuscript.

\section{Additional information}

Supplementary Information accompanies this paper at http://www.nature.com/ naturecommunications

Competing financial interests: The authors declare no competing financial interests.

Reprints and permission information is available online at http://npg.nature.com/ reprintsandpermissions/

How to cite this article: Hong, J. et al. Exploring atomic defects in molybdenum disulphide monolayers. Nat. Commun. 6:6293 doi: 10.1038/ncomms7293 (2015).

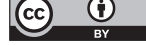

This work is licensed under a Creative Commons Attribution 4.0 International License. The images or other third party material in this article are included in the article's Creative Commons license, unless indicated otherwise in the credit line; if the material is not included under the Creative Commons license, users will need to obtain permission from the license holder to reproduce the material. To view a copy of this license, visit http://creativecommons.org/licenses/by/4.0/ 